\title{
Medindo Eficiência em Desenvolvimento de Sistemas
}

\author{
Antonio Allen Meireles Alcantara \\ Programa de Engenharia de Produção - COPPE/UFRJ \\ allen@br-petrobras.com.br \\ Annibal Parracho Sant'Anna \\ Departamento de Engenharia de Produção - UFF \\ Rua Passo da Pátria, 156 24210-240 Niterói-RJ \\ annibal@ruralri.com.br
}

Estudamos neste trabalho as possibilidades de utilização da Análise de Encapsulamento de Dados (DEA) na avaliação da produtividade no desenvolvimento de sistemas. Apontamos um conjunto de variáveis de entrada, que medem os volumes de recursos utilizados, e de saída, que medem o volume da produção resultante. Estudamos relações entre essas variáveis de modo a reduzi-las em número e comparamos os resultados da aplicação de diferentes modelos de DEA. Os resultados obtidos indicam que esta metodologia pode ser aplicada eficientemente para classificar equipes operando em ambientes comparáveis.

Palavras-chave: Análise de Encapsulamento de Dados, medição de eficiência, avaliação cruzada

We study here the possibility of application of data Envelopment Analysis (DEA) to the evaluation of productivity in computational systems development. We choose input and output variables for this process and study the relations between them that may allow to simplify the model. We compare the results of application of different DEA models. The results obtained show that cross evaluation can be successfully applied to rank system development teams working in similar environments.

\section{INTRODUÇÃO}

O número de sistemas vem crescendo

exponencialmente. Dados, como os de Jones (1966), permitem estimar que este número dobra em um prazo de cinco a dez anos. Por outro lado, nossa capacidade de gerenciar o processo de desenvolvimento e manutenção não progrediu em eficiência, comparativamente, por exemplo, à capacidade tecnológica de construir grandes computadores. Notadamente, os progressos alcançados na melhoria de produtividade nestas áreas são devidos, quase que exclusivamente, ao aparecimento de ferramentas CASE, linguagens de alto nivel e metodologias criadas para desenvolvimento $e$ manutenção. O que se observá é que foi insuficiente o esforço despendido para identificar, medir e interpretar fatores que podem interferir na produtividade dentro do ambiente onde atuam programadores $e$ analistas.

Para avaliar a eficiência no desenvolvimento de sistemas por diferentes equipes, precisamos, principalmente, ser capazes de combinar diferentes formas de medir resultados obtidos em diferentes fases do processo em que diferentes aspectos são mais importantes. A Análise de Encapsulamento de Dados (DEA) permite combinar indicadores gerando padrões de desempenho a partir dos próprios desempenhos observados. O objetivo deste artigo é explorar esta possibilidade.

THADHANI (1984) investiga os fatores que influenciam a produtividade durante o desenvolvimento de aplicativos computacionais. Dentre os principais fatores encontrados nesse estudo, destacam-se: a qualidade das ferramentas utilizadas, a linguagem implementada, a adequação às técnicas de programação, a complexidade do sistema e a habilidade da equipe. Estes fatores são preponderantes no resultado final da produtividade alcançada, porém, sob condições similares de trabalho sempre encontraremos diferenças de desempenho que nos indicarão algumas variáveis, controláveis ou não, interferindo na produtividade das equipes. Isto sugere procurar conjuntos de equipes suficientemente homogêneos para que comparações façam sentido e tentar identificar diferenças entre elas.

O primeiro obstáculo para o estudo da produtividade de equipes desenvolvedoras de sistemas está na dificuldade de medir objetivamente o produto do trabalho realizado por essas equipes, ou seja, o tamanho do software. A 
produtividade deve ser medida pela razão entre produto efetivamente disponibilizado e esforço despendido. A métrica baseada em linhas de código, uma das mais difundidas, não é indicada para estudos de produtividade, já que linhas codificadas não podem ser encaradas nem como bens e nem como serviços prestados ao usuário final. Nos últimos anos, a metodologia de Análise de Pontos Funcionais (FPA), como padronizada pelo International Function Point Users Group (IFPUG) (1999), vem-se revelando capaz de medir a funcionalidade da aplicação que beneficia o usuário final. $O$ uso desta metodologia para medir o tamanho dos sistemas é que vai viabilizar a abordagem de comparação de produtividade aqui desenvolvida.

A FPA, criada para dimensionar sistemas tendo como ponto de partida o aspecto exterior visível ao usuário, atende a 5 pontos essenciais:

- trata os aspectos externos do software

- trata aspectos considerados importantes para o usuário

- pode ser aplicada durante o desenvolvimento de um projeto

- pode ser utilizada para cálculo de produtividade econômica

- é independente de código fonte ou linguagem

Assim, ao longo deste trabalho, utilizaremos como medida de complexidade de software a quantidade de pontos funcionais (FP's). A metodologia padrão de contagem está descrita no Manual de Práticas de Contagem do IFPUG.

Para compor os indicadores usamos a DEA. Esta metodologia permite comparar um conjunto de unidades de produção constituído por equipes desenvolvedoras de Sistemas de Informação operando sob condições "similares" de trabalho. Pela sua capacidade de manusear inputs e outputs de natureza multidimensional, DEA se mostra adequada para analisar o desempenho dessas equipes. Uma completa descrição da metodologia e ampla lista de aplicações recentes de DEA se encontram em Charnes, Cooper, Lewin e Seiford (1994) e Cooper, Seiford e Tone (1999), por exemplo.

$D E A$ não tem sido usada diretamente na comparação de tomadas de decisão no desenvolvimento de software.
Um artigo básico em DEA que abre perspectivas nessa direção é o de Banker, Datar e Kemerer (1991). Depois disso, encontramos uma ampla discussão dos problemas da área em Reese (1997), mas nenhuma aplicação efetiva. Conforme veremos nas seções seguintes, um programa de avaliação de eficiência utilizando esta metodologia não necessita a priori formulação de hipóteses sobre formas funcionais como nos modelos paramétricos tradicionais. Exige, por outro lado, uma análise minuciosa de critérios (alguns subjetivos) a serem respeitados durante as diversas fases da aplicação da metodologia, como seleção das unidades tomadoras de decisão (DMU's), seleção dos fatores, restrição dos multiplicadores e formulação de modelos.

Um passo importante antes de começarmos a construir um modelo de medição é termos uma definição clara dos objetivos a serem alcançados com o estudo. Os objetivos mais comuns encontrados em estudos de DEA são, segundo Norman e Stoker (1991):

- aumento de vendas

- redução de custos

- melhoria de efícácia

- identificação de bons desempenhos

- identificação de baixos desempenhos

- investigação de estruturas organizacionais

- racionalização

- premiar desempenho

Alguns dos maiores benefícios que a administração alcança ao introduzir medidas de desempenho na organização são: maior conhecimento dos processos realizados dentro das unidades, identificação de onde $e$ quando a área gerencial deve tomar ações para melhorar desempenho e ainda, criação de um ambiente mais propicio para um controle mais eficiente da qualidade.

Este trabalho estuda a produção de sistemas conduzida em uma Gerência de Informática. O que se busca identificar são os níveis de produtividade e qualidade do produto final gerado pelas equipes. Mais especificamente, a administração deseja respostas a algumas questões consideradas relevantes para a montagem das diversas equipes na área de desenvolvimento: quanto a motivação está influenciando 
a produtividade das equipes? As equipes com mais tempo de experiência respondem positivamente a este diferencial? Qual o critério mais eficiente na distribuição de programadores $e$ analistas $\mathrm{em}$ um projeto?

$\mathrm{Na}$ Seção seguinte, discutimos estes objetivos, identificamos os fatores relevantes para a produtividade $e$ selecionamos as variáveis para a análise. $\mathrm{Na}$ Seção 3, aplicamos a metodologia desenvolvida a um caso concreto. $\mathrm{Na}$ Seção 4, conclusões são extraídas da análise do caso estudado.

\section{METODOLOGIA DE COMPARAÇÃO DE DESEMPENHONO DESENVOLVIMENTO DE SISTEMAS}

A sistemática aqui construida para estudo de análise de eficiência terá como objetivo final apresentar a uma Gerência de Informática um instrumento que auxilie na tomada de decisão, oferecendo ao gerente alternativas de ações que aumentem a produtividade das equipes $e$ melhoria na qualidade dos serviços prestados. Para aplicar DEA, as unidades que comparamos devem desempenhar as mesmas tarefas, com recursos tecnológicos comparáveis, tendo objetivos similares. Além disso, os fatores que afetam seu desempenho devem ser os mesmos, exceto por diferenças em intensidade e magnitude.

Seguindo este raciocínio, alguns critérios iniciais devem ser colocados para seleção das DMU's no contexto do presente trabalho. Conforme descrito anteriormente, fatores como ferramentas utilizadas, linguagem implementada, técnicas de programação, complexidade dos sistemas e habilidade da equipe podem produzir unidades heterogêneas que não devem ser grupados num mesmo estudo. Assim, um cuidado inicial, que conduz a uma padronização na maior parte dos fatores referidos, deve ser o de selecionar projetos sendo desenvolvidos sob uma mesma plataforma (mainframe).

As linguagens de programação utilizadas afetam a produtividade. Podemos, porém, desprezar essas diferenças, pois a variação na produtividade não é muito acentuada. Segundo JONES (1996), o esforço de codificação corresponde a não mais de $20 \%$ do esforço total.

Quanto à complexidade, sabe-se que estudos de produtividade realizados levando em consideração pequenos projetos costumam apresentar resultados distorcidos por dois fatores: habilidades individuais $e$ experiência prévia no sistema. Por isso, é conveniente estabelecer uma faixa de variação para o total de $F P^{\prime}$ s. Outro ponto a que devemos estar atentos é o período em que foram desenvolvidos os projetos. Épocas muito distantes entre si poderiam encobrir uma série de atualizações tecnológicas ou de mudança de processos internos ocorridos na Gerência.

A introdução de DEA como uma ferramenta gerencial implica sempre na criação de Sistemas de Coleta de Dados que serão os alimentadores da análise.

Geralmente, ao selecionarmos os fatores, é verificado que os dados não existem ou são imprecisos. Uma boa prática, ao introduzir DEA, é, após a escolha dos fatores, iniciar imediatamente a coleta de dados.

A princípio, a lista inicial de fatores (inputs e outputs) deve ser tão grande quanto possível. Porém, na ausência de um grande número de DMU'S, a utilização de um grande número de fatores acarreta um menor poder discriminante das unidades, tendendo a apresentar um número excessivo de unidades classificadas como plenamente eficientes. Assim, precisamos ter procedimentos de seleção que permitam introduzir no modelo somente um número limitado de fatores.

Todo recurso usado pela unidade deve ser incluído como input. Ainda que recursos de diferente origem possam ser agregados para diminuir a dimensão do vetor de variáveis, é importante destacar fatores de natureza diversa: o volume de insumos propriamente ditos, o tempo de sua utilização, a qualidade dos mesmos, a motivação na sua utilização, etc.

Por outro lado, a unidade converte recursos para produzir outputs de interesse, de modo que estes últimos devem representar quantidades de produtos ou serviços com níveis aceitáveis de qualidade. Um importante elemento na medição da produtividade é a relação entre os níveis de qualidade dos produtos oferecidos pelas diferentes unidades. $O$ conceito de qualidade que usaremos aqui é de qualidade operacional, se o sistema opera sem problemas de parada e sem produzir resultados incorretos.

Os fatores podem ser controláveis (fatores gerenciais) ou não-controláveis pelas DMU's (fatores ambientais).

Podem, ainda, ser quantitativos (prontamente disponíveis em termos numéricos) ou qualitativos. Neste segundo tipo de fator, usualmente representando 
satisfação do usuário e medido através de pesquisas de opinião, um procedimento de quantificação deve ser realizado para permitir o uso de DEA

\subsection{Outputs inicialmente Considerados}

Conforme destacado na seção anterior, os outputs devem representar a quantidade de produtos ou serviços gerados e avaliados por um indicador de qualidade. No nosso modelo de produção de sistemas teremos os seguintes fatores como output:

- números de FP's produzidos pela unidade

- quantidade de defeitos

Enquanto o primeiro é um output eminentemente quantitativo, o segundo é um fator qualitativo, indicando a capacidade do sistema entrar em produção sem ficar inoperante e apresentar as funções capacitadas e corretas. Adotamos como critério de tempo para registro de defeitos os primeiros 6 meses do sistema em produção. Para avaliarmos a magnitude das falhas encontradas, as mesmas devem ser ponderadas segundo a Tabela I.

Assim, utilizamos como indicador das falhas encontradas no sistema a seguinte expressão:

Nível de Falhas $=1 / \sum_{i=1}^{4} p_{i} x_{i}$

onde

pi $=$ peso do i-ésimo tipo de falha,

$x i=$ quantidade do i-ésimo tipo de falha.
A fórmula acima produz valores coerentes com o tipo de fator sob análise (output): valores maiores indicam melhor qualidade dos sistemas.

\subsection{Inputs inicialmente Considerados}

- Custos do Projeto

É uma medida de recurso e reflete o custo de desenvolvimento do projeto. Inclui o custo financeiro total da equipe.

- Tempo de execução do projeto

É medido em homens * meses. É inicializado na fase de definição do sistema indo até fase de teste antes da entrega para a produção.

- Experiência da equipe.

Tempo médio em anos na atividade de PD dos integrantes da equipe.

- Motivação Pessoal

Motivação pessoal na área de informática tem sido objeto de vários estudos. Utilizamos, seguindo Kemayel, Mili e Kedemi (1991), o questionário no Anexo I para avaliarmos 16 itens que refletem motivação pessoal. As respostas utilizam uma escala de 1 a 5 onde a atribuição dos valores segue a ordem das respostas. $O$ menor valor reflete maior motivação pessoal. Como indicador do input consideramos o score médio dos integrantes da equipe.

\section{Aplicação}

Aplicamos a metodologia desenvolvida na Seção anterior à avaliação do desempenho na Gerência de Informática da Petrobrás Distribuidora no Rio de Janeiro. As

\section{Pesos}

\begin{tabular}{clc}
\hline Código de Severidade & \multicolumn{1}{c}{ Tipo de Falha } & Peso \\
\hline 1 & Sistema ou Programas inoperante & $(0,05)$ \\
2 & Funções maiores incapacitadas & $(0,04)$ \\
3 & Funções menores incapacitadas & $(0,02)$ \\
4 & Falha Superficial & $(0,01)$ \\
\hline
\end{tabular}


medidas selecionadas são baseadas em dados iniciais alcançados após a implantação de Sistemas Coletores e apresentadas na Tabela II, referentes a 12 projetos de desenvolvimento onde os dados são carregados basicamente da Ficha Financeira (custos financeiros da equipe) e do Sistema de Informação de Projetos operado por cada líder de projeto (número de FP's produzidos, falhas ocorridas, tempo de execução do projeto e experiência da equipe).

As linguagens de programação utilizadas nos projetos em estudo são COBOL e CSP. Apesar de serem do mesmo nível, sabemos que a escolha dentre estas linguagens afeta a produtividade. Como estabelecido na Seção anterior, consideramos irrelevantes as diferenças dai decorrentes. Para garantir a homogeneidade quanto à complexidade, foram considerados, apenas, projetos tendo a quantidade de pontos funcionais produzidos variando entre 120 e 2000.

A opção pelo conjunto de cinco variáveis apresentadas acima é puramente baseada na experiência de especialistas.
Consideramos inicialmente as seis variáveis propostas na seção anterior, mas, a alta correlação do tempo de execução com o custo do projeto no conjunto de dados analisado (coeficiente de correlação de 0,96) nos levou a excluí-la da análise. $O$ efeito dessa exclusão foi um pequeno aumento do poder de discirminação do modelo. É importante notar que, como o número de DMU's é relativamente pequeno comparado ao número de variáveis, torna-se interessante reduzir ao máximo o número de fatores. De fato, um valor pequeno para a relação da quantidade de DMU's para a quantidade de variáveis resulta em apontar muitas unidades como eficientes.

\subsection{Análise de Eficiência}

Os scores de eficiência e os respectivos conjuntos de referência (peer groups) são dados na Tabela III. Como pode ser visto, os resultados são bastante similares utilizando seja o modelo de Banker, Cooper e Charnes (1984), o chamado modelo BCC, corespondente à hipótese de efeitos constantes de esclala, seja o modelo

Tabela II

Dados

\section{Outputs}

Inputs

\begin{tabular}{cccccc}
\hline $\begin{array}{c}\text { DM } \\
\mathbf{U}\end{array}$ & $\begin{array}{c}\text { Pontos } \\
\text { Funcionais }\end{array}$ & $\begin{array}{c}\text { Nível de } \\
\text { Falhas }\end{array}$ & $\begin{array}{c}\text { Custos } \\
\text { RS }\end{array}$ & $\begin{array}{c}\text { Experiência da } \\
\text { Equipe (anos) }\end{array}$ & $\begin{array}{c}\text { Motivação } \\
\text { Pessoal }\end{array}$ \\
\hline 1 & 552 & 3,57 & 91.000 & 8,0 & 56 \\
2 & 131 & 2,00 & 15.900 & 1,5 & 42 \\
3 & 788 & 2,27 & 85.200 & 7,5 & 50 \\
4 & 379 & 4,16 & 45.500 & 3,0 & 38 \\
5 & 355 & 2,56 & 59.400 & 5,0 & 46 \\
6 & 148 & 6,25 & 40.000 & 7,0 & 49 \\
7 & 370 & 2,43 & 50.400 & 3,5 & 39 \\
8 & 273 & 11,11 & 64.800 & 6,0 & 41 \\
9 & 326 & 1,01 & 55.200 & 5,0 & 38 \\
10 & 1.115 & 2,70 & 126.000 & 9,5 & 51 \\
11 & 132 & 4,76 & 22.400 & 3,0 & 29 \\
12 & 201 & 1,85 & 55.000 & 4,0 & 31 \\
\hline
\end{tabular}


de Charnes, Cooper e Rhodes (1978), o chamado modelo CCR, que admite efeitos variáveis de escala. Isto sugere existir um fraco efeito de escala nos dados. A ausência da restrição de convexidade no modelo $\mathbf{C C R}$ produz uma discriminação um pouco maior nos resultados (observar médias). A grande quantidade de unidades eficientes (7 nos dois modelos) é resultante da baixa relação DMUs/fatores. Conforme Golany e Roll (1989), um critério razoável para eliminar um fator se obtém pela análise da matriz de correlação dos fatores. A idéia é eliminar fatores que possuam mesmo conteúdo de informação fornecido por outros.
Ambos os modelos foram ajustados sob a ótica de minimização do uso de inputs, tendo em vista que, em geral, a dimensão do produto é dada e os ganhos de produtividade são gerados pela redução de inputs. A principal vantagem de adotar a ótica da minimização é, de fato, de ordem prática, a de permitir medir a eficiência de cada equipe pelo percentual dos recursos efetivamente empregados que seria suficiente para atingir a produção apresentada.

Neste estágio, uma simples contagem na frequência com que as unidades aparecem no conjunto referência é um

Tabela III.

DEA com 2 outputs e 3 inputs

\begin{tabular}{ccccc}
\hline \multirow{2}{*}{ DMU } & \multicolumn{2}{c}{ CCR } & \multicolumn{2}{c}{ BCC } \\
& (Retorno Constante de Escala) & (Retorno Variável de Escala) \\
\hline & Eficiência (\%) & Conjunto & Eficiência & Conjunto \\
1 & & Referência & $(\%)$ & Referência \\
2 & 70,56 & 3,4 e 10 & 71,49 & $3,4,10$ e 11 \\
3 & 100,00 & 2 & 100,00 & 2 \\
4 & 100,00 & 3 & 100,00 & 3 \\
5 & 100,00 & 4 & 100,00 & 4 \\
6 & 68,35 & 2,3 e 4 & 76,82 & 4,10 e 11 \\
7 & 75,05 & 8 & 80,87 & 8 e 11 \\
8 & 86,14 & 4 e 10 & 94,85 & 4,10 e 11 \\
9 & 100,00 & 8 & 100,00 & 8 \\
10 & 63,99 & 2 e 3 & 87,74 & 10 e 11 \\
11 & 100,00 & 10 & 100,00 & 10 \\
12 & 100,00 & 11 & 100,00 & 11 \\
\hline Eficiência & 48,58 & 4 e 8 & 98,53 & 10 \\
Média & 84,38 & & 92,52 & \\
\hline Ineficiência & 68,77 & & & \\
Média & & & 85,05 & \\
\hline
\end{tabular}


indicador para discriminarmos os "melhores" dentre todos os eficientes. Esta frequência retrata como uma DMU eficiente aparece como um padrão de avaliação das unidades ineficientes. Assim, um critério inicial e simples para ordenarmos as DMU's eficientes seria pela quantidade de vezes que ela aparece no conjunto referência das unidades ineficientes. A unidade 4, fazendo parte do conjunto referencia das unidades 1,5,7 e 12, seria escolhida a "melhor" dentre todas as eficientes.

Um ponto importante a ser considerado na análise dos resultados, e não contemplado na Tabela III, é a distribuição dos pesos que cada DMU especificou para alcançar sua taxa de eficiência. Na sua formulação básica, DEA permite quase que total flexibilidade na seleção dos pesos dos fatores (comumente anunciado como uma vantagem da metodologia) e este fato deve ser analisado com muito cuidado, pois poderá esconder resultados indesejáveis (Wong e Bealey, 1990). Os pesos representam um valor relativo que proporciona o melhor score possivel para uma determinadada DMU, além disso, esse sistema de peso deve garantir que nenhuma DMU alcance um score de eficiência acima da unidade. Nestas condições, é comum algum fator de menor relevância adquirir um peso relativamente alto, gerando uma unidade eficiente basicamente à custa desse fator, tendo, por outro lado, peso nulo para fatores mais relevantes. Isto é inaceitável, do ponto de vista prático.

A Tabela IV fornece os pesos relativos das variáveis. Uma técnica que minimiza a dependência de determinado score alcançado por uma DMU dos sistemas de pesos é a Avaliação Cruzada (Cross Evaluating) a ser abordada na próxima subseção.

\section{Pesos das Variáveis}

\begin{tabular}{ccccccc}
\hline \multicolumn{2}{c}{ Outputs } & & & Inputs & \\
\hline & & & & & Experiência \\
DMU & Eficiência(\%) & Pontos & Motivação \\
& & Funcionais & Nivel Falhas & Custos( R\$) & $\begin{array}{c}\text { Equipe } \\
\text { (anos) }\end{array}$ & Pessoal \\
\hline 1 & 70,56 & 86 & 14 & 82 & 0 & 18 \\
2 & 100,00 & 81 & 19 & 94 & 0 & 6 \\
3 & 100,00 & 96 & 4 & 99 & 0 & 1 \\
4 & 100,00 & 86 & 14 & 98 & 0 & 2 \\
5 & 68,35 & 90 & 10 & 98 & 0 & 2 \\
6 & 75,05 & 0 & 100 & 64 & 0 & 36 \\
7 & 86,14 & 55 & 45 & 0 & 100 & 0 \\
8 & 100,00 & 39 & 61 & 70 & 0 & 30 \\
9 & 63,99 & 97 & 3 & 100 & 0 & 0 \\
10 & 100,00 & 100 & 0 & 0 & 0 & 100 \\
11 & 100,00 & 51 & 49 & 100 & 0 & 0 \\
\hline 12 & 48,58 & 67 & 33 & 0 & 53 & 47 \\
\hline
\end{tabular}


PRODUCAC

\subsection{Avaliação Cruzada (Cross Evaluation)}

Na subseção anterior, utilizando o critério de correlação para seleção de fatores, pouco foi conseguido no sentido de aumentar o poder de discriminação das unidades. Uma boa maneira de avançarmos nessa direção, principalmente fazendo uma discriminação entre todas as DMU's eficientes, além de contornar os problemas devidos à grande quantidade de pesos zero atribuídos a muitas variáveis consideradas relevantes, é a utilização da técnica de Avaliação Cruzada (Cross Evaluating), desenvolvida por Sexton et al. (1986).

Esta técnica cria uma Matriz de Eficiência Cruzada contendo informações de todas as DMU's quanto a distribuição de sua eficiência relativa às outras unidades. Para isso, para cada DMU, calculamos sua eficiência utilizando os pesos ótimos das outras unidades. O resultado será uma visualização de como uma determinada unidade alcança sua eficiência segundo ela mesmo (self-appraisal) e também com referência aos critérios ótimos para cada uma das demais (peer-appraisal).

$O$ método pode ser resumidamente descrito assim. Denotamos por $\mathrm{E}_{\mathrm{ks}}=\Sigma \mathrm{O}_{\mathrm{sy}} \mathrm{v}_{\mathrm{ky}} / \Sigma \mathrm{I}_{\mathrm{sx}} \mathrm{u}_{\mathrm{kx}}$ a eficiência cruzada da DMU s utilizando o sistema de pesos ótimos da $\mathbf{D M U} k$, obtido a partir da formulação padrão CCR. Nesta expressão, o primeiro somatório é indexado pelo indice y que varia ao longo dos outputs $(O)$, enquanto o segundo é indexado pelo índice $x$ que varia ao longo dos inputs (I). Notamos, facilmente, que $E_{k h}$ é a eficiência da DMU $k$ utilizando os próprios pesos, resultando na eficiência até agora calculada (chamada eficiência

padrão).

Desde que a Avaliação Cruzada utiliza os pesos $u_{k x e}$ e v ${ }_{k y^{\prime}}$ calculados a partir do modelo DEA padrão, e como na solução do problema de programação linear padrão podemos encontrar múltiplas soluções ótimas, ao efetuarmos o cálculo de $E_{k s}$ poderíamos obter resultados bastante diferentes. Para contornarmos esta dificuldade, procedemos da seguinte maneira, conforme Doyle e Grenn (1994): calculamos uma solução DEA padrão ótima para cada unidade $K\left(E_{k k}\right)$ e depois utilizamos uma função objetivo secundária que minimize a soma das eficiências cruzadas das demais unidades. Esta formulação, utilizando minimização das eficiências das demais unidades, denotada $B_{1}$, é chamada agressiva. A alternativa de maximizá-las é chamada de benevolente.
Com o objetivo de acentuar a discriminação entre as eficiências, optamos pela formulação agressiva. $O$ problema de programação linear a ser, então, usado terá a seguinte forma:

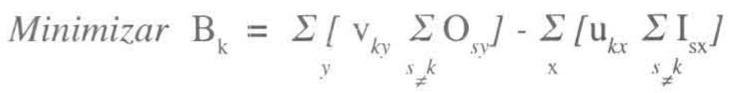

Sujeito a :

$\sum \mathrm{I}_{k \mathrm{x}} \mathrm{u}_{k \mathrm{x}}=1$

$\sum \mathrm{O}_{k y} \mathrm{v}_{k y}-\mathrm{E}_{k k} \sum \mathrm{Ikx} \cdot \mathrm{u}_{k x}=0$

$\sum \mathrm{O}_{k y} \mathrm{v}_{k y}-\sum \mathrm{I}_{\mathrm{x}} \mathrm{u}_{k x} \leq 0 \quad \mathrm{~s} \neq \mathrm{k} \quad \mathrm{u}_{\mathrm{kx}} e \mathrm{v}_{\mathrm{ky}} \geq 0$

Primeiro, resolvemos o problema de programação linear acima, encontrando o esquema de pesos que serão utilizados para obter as eficiências cruzadas $\left(E_{k}\right)$, os elementos integrantes da Matriz de Eficiência Cruzada. Um critério para depois classificarmos as DMU's apresentado em Estellita Lins e Meza (1999) é $\mathrm{e}_{\mathrm{k}}=1 / \mathrm{n} \Sigma$ $\mathrm{E}_{\mathrm{sk}}$, a eficiência cruzada média da DMU k. As Tabelas VI e VII apresentam esses cálculos. Estes resultados são um bom complemento à eficiência padrão para distinguir entre várias $\mathrm{DMU}$ 's eficientes, possibilitando obter uma ordenação significativa.

Podemos comparar a Tabela VII com a Tabela III e verificar a disponibilidade de informação mais adequada na Tabela VII. As seis equipes que obtiveram $100 \%$ de eficiência no modelo CCR padrão foram classificadas nos seis primeiros lugares utilizando a eficiência cruzada média, porém agora apresentam niveis discriminantes.

Finalmente, devemos identificar as equipes com alto grau de mudança da eficiência padrão para a eficiência média cruzada, denominadas de mavericks (Doyle e Green, 1984), calculados por: $M_{k}=\left(E_{k k}-e_{k}\right) / e_{k}$.

Um alto valor de $M_{k}$ sugere uma DMU com uma diferença relativa acentuada entre a eficiência padrão $e$ eficiência cruzada, ou seja, o esquema de peso utilizado por ela não é realista nem está em consonância com as outras DMU's. A Tabela VIII apresenta os valores $M_{k} . A$ pequena variação registrada apenas ajuda a confirmar a classificação estabelecida. 
Tabela V.

Pesos da Formulação Agressiva $B_{k}$

\begin{tabular}{ccc|ccc}
\hline \multicolumn{3}{c|}{ Outputs } & \multicolumn{3}{c}{ Inputs } \\
\hline DMU & Function Points & Nivel Falhas & Custos R\$ & $\begin{array}{c}\text { Experiência } \\
\text { Equipe (anos) }\end{array}$ & $\begin{array}{c}\text { Motivação } \\
\text { Pessoal }\end{array}$ \\
\hline 1 & 0,001098 & 0,027872 & 0,00000902 & 0 & 0,003208 \\
2 & 0,004076 & 0,233017 & 0,00003750 & 0,268831 & \\
3 & 0,001269 & 0 & 0,00001010 & 0 & 0,002785 \\
4 & 0,002639 & 0 & 0 & 0,342293 & 0 \\
5 & 0,0001736 & 0,026196 & 0,0000165 & 0,0000201 & 0,000411 \\
6 & 0 & 0,12008 & 0,0000159 & 0,0000191 & 0,007447 \\
7 & 0,002328 & 0 & 0,000011 & 0,127901 & 0,00000176 \\
8 & 0 & 0,090009 & 0 & 0 & 0,02439 \\
9 & 0,001903 & 0,019379 & 0,0000181 & 0,00000841 & 0 \\
10 & 0,000897 & 0 & 0 & 0,099349 & 0,001102 \\
11 & 0 & 0,210084 & 0,0000446 & 0 & 0 \\
12 & 0,001605 & 0,0088231 & 0 & 133396 & 0,015394 \\
\hline
\end{tabular}

Tabela VI

Matriz de Eficiências Cruzadas (Formulação Agressiva BK)

\begin{tabular}{|c|c|c|c|c|c|c|c|c|c|c|c|}
\hline 1 & 2 & 3 & 4 & 5 & $\overline{6}$ & 7 & 8 & 9 & 10 & 11 & 12 \\
\hline 0,7052 & 0,7175 & 0,9995 & 0,9995 & 0,6748 & 0,6500 & 0,8176 & 0,8511 & 0,6229 & 0,9995 & 0,9407 & 0,4571 \\
\hline 539 & 1,0004 & 0,7178 & 1,0005 & 0,5721 & 0,6090 & 0,7327 & 0,9155 & 0,4581 & 0,7108 & 1,0004 & 0,3984 \\
\hline 0,6515 & 0,5989 & 1,0002 & 8506 & 0,6187 & 0,3475 & 0,7601 & 0,4506 & 0,6236 & 1,0002 & 0,5456 & 0,3974 \\
\hline 0,5319 & 0,6733 & 0,8100 & 0,9740 & 0,5473 & 0,1630 & 0,8150 & 0,3507 & 0,5026 & 0,9048 & 0,3392 & 0,3874 \\
\hline 0,6898 & 1,0005 & $1,000 t$ & 1,0006 & 0,6839 & 0,6183 & 0,8 & 42 & 0,6393 & 53 & 0,9273 & 0,4318 \\
\hline 0,2299 & 0,4246 & 0,1578 & 0,4963 & 0,2388 & 0,7497 & 0,2672 & 0,9987 & 0,1044 & 0,1360 & 0,9989 & 0,2009 \\
\hline 0,6348 & 0,8313 & 0,9672 & 0,9977 & 0,6391 & 0,2580 & 0,8597 & 0,4293 & 0,6087 & 0,9979 & 0,4876 & 0,4190 \\
\hline 0,2352 & 0,1757 & 0,1675 & 0,4040 & 0,2053 & 707 & 299 & 1,0000 & 1,0000 & 0,0980 & 0,1953 & 0,6057 \\
\hline 0,6797 & 1,0008 & 1,0008 & 9736 & 0,6744 & 562 & 0,8234 & 0,6264 & 0,6404 & 32 & 0,8470 & 0,4 \\
\hline 0,5780 & 0,6016 & 0,8833 & 1,0001 & 0,5816 & 0,1771 & 0,8494 & 0,3818 & 0,5429 & 1,0001 & 0,3587 & 0,4177 \\
\hline 0,1847 & 0,5925 & 0,1255 & 0,4306 & 0,2030 & 0,7360 & 0,2271 & 0,8076 & 0,0861 & 0,1009 & 1,0009 & 0,1584 \\
\hline 0,6224 & 0,4567 & 0,8276 & 0,9900 & 0,5786 & 0,4673 & 0,7573 & 0,9908 & 0,4891 & 0,9880 & 0,7463 & 0,4806 \\
\hline 0,52 & 0,6728 & 0,7215 & 0,8431 & 0,5181 & 0,4836 & 0,6643 & 0,7089 & 0,5265 & 0,7371 & 0,699 & 0,3979 \\
\hline
\end{tabular}


Tabela VII.

Ranking das Equipes utilizando Experiência Cruzada.

\begin{tabular}{ccccccccccccc}
\hline 1 & 1 & 2 & $\vdots$ & 4 & 5 & 6 & 7 & 8 & 9 & 10 & 11 & 12 \\
\hline 4 & 10 & $\vdots$ & 8 & 11 & 2 & 7 & 9 & 1 & 5 & 6 & 12 \\
0,8431 & 0,7371 & 0,7215 & 0,7089 & 0,699 & 0,6728 & 0,6643 & 0,5265 & 0,5248 & 0,5181 & 0,4836 & 0,3979 \\
\hline
\end{tabular}

Tabela VIII.

Classificação de DMU's em valores de $M_{k}$

\begin{tabular}{cccc}
\hline DMU & $\mathrm{E}_{\mathrm{kk}}$ & $\mathrm{e}_{\mathrm{k}}$ & $\mathrm{M}_{\mathrm{k}}$ \\
\hline 6 & 0,7505 & 0,4836 & 0,55 \\
2 & 1 & 0,6728 & 0,48 \\
11 & 1 & 0,699 & 0,43 \\
8 & 1 & 0,7089 & 0,40 \\
3 & 1 & 0,7215 & 0,38 \\
10 & 1 & 0,7371 & 0,35 \\
1 & 0,7056 & 0,5248 & 0,34 \\
5 & 0,6835 & 0,5181 & 0,31 \\
7 & 0,8614 & 0,6643 & 0,29 \\
12 & 0,4858 & 0,3979 & 0,22 \\
9 & 0,6399 & 0,5248 & 0,21 \\
4 & 1 & 0,8431 & 0,18 \\
\hline
\end{tabular}

\section{CONCLUSÃO}

Diversas metas foram atingidas ao longo deste trabalho. Primeiro, foi realizada uma seleção de variáveis para medição do desenvolvimento de sistemas, tanto do ponto de vista dos recursos utilizados quanto do ponto de vista dos resultados apresentados. Estas variáveis medem, do lado dos recursos: custos, experiência da equipe e motivação; do lado dos produtos, número de pontos funcionais produzidos e ausência de defeitos.

Em especial, ficou demonstrado como a técnica de Análise de Pontos Funcionais pode ser empregada pra quantificar efetivamente a produção. A seguir, ficou demonstrado como a técnica de Análise de Encapsulamento de Dados pode ser empregada, para extrair, das medidas de recursos e produtos consideradas, uma comparação entre os desempenhos de diversas equipes. Neste ponto, também, um detalhe merece especial atenção, qual seja o uso da técnica de avaliações cruzadas para permitir estabelecer uma classificação estrita em um conjunto de equipes, das quais cerca de metade era considerada igualmente eficiente quando se levavam em conta apenas os critérios de ponderação preferidos de cada uma.

\section{Referências}

Banker, R. D., Charnes, A. e Cooper, W. W. (1984). Some Models for Estimating Technical and Scale Inefficiencies in DEA, Management Science, 30, pp 1078-1092. 
Banker, R. D., Datar, S. M. e Kemerer, C. F. (1991). A Model to Evaluate Variables Impacting the Productivity of Software Maintenance Projects. Management Science, 37, pp. 1-18.

Charnes, A., Cooper, W. W. e Rhodes, E. (1978). Measuring the Efficiency of Decision Making Units, European Journal of Operational Research, 2, pp. 429-444.

Charnes, A., Cooper, W. W., Lewin, A. Y. e Seiford, L. M. Data Envelopment Analysis: Theory, Methodology and Applications, Kluwer Academic Publishers, Dordrecht, 1994.

Cooper, W. W., Seiford, L. M. e Tone, K. (1999). Data Envelopment Analysis: A Comprehensive Text with Models, Applications, References and DEA-SOLVER Software, Kluwer Academic Publishers, Dordrecht, 1999.

Doyle, J. and Green, R. (1994), Efficiency and Cross-Efficiency in DEA : Derivations, Meanings and Uses, Journal Operational Research Society, vol. 45, n. 5, pp. 567-578.

Estellita Lins, M. e Meza, L. A. Análise Envoltória de Dados, COPPE-UFRJ, Rio de Janeiro, 1999.

Golany, B. and Roll, Y. (1989), An application procedure for DEA, OMEGA, vol. 17, n.3 pp. 237-250

International Function Point Users Group (IFPUG), Function Point Counting Practices Manual, Release 4.1, 1999.

Jones, C. , Applied Software Measurement: Assuring Productivity and Quality, Mc Graw-Hill Book Co., N. York, 1996.

Kemayel, L., Mili, A and Quederni, I. (1991), Controllable Factors for Programmer Productivity : A Statistical Study, Journal Systems Software, 16, pp. 151-163

Norman, M. and Stoker, B., Data Envelopment Analysis:The assessment of Performance, John Wiley \& Sons, New York, 1991.

Reese, D. N. Applications of DEA to Software Engineering Management. U. of Tornoto Ph. D. thesis, 1997.

Sexton, T. R., Silkman, H. R. and Hogan, A. J., Data Envelopment Analysis: Critique and extensions, in Measuring Efficiency: an assessment of Data Envelopment Analysis. Jossey-Bass, San Francisco, 1986.

Thadhani, A. (1984), Factors Affecting Programmer Productivity During Application Development, IBM System Journal, Vol. 23, n 1, pp. 19 -35.

Wong, Y. and Beasley, J. (1990), Restricting Weight Flexibility in Data Envelopment Analysis, Journal Operational Research Society. vol. 41 n. 9, pp. 829-835. 


\section{Anexo I}

\section{Motivação Pessoal.}

1. Seu trabalho oferece oportunidades para realizações técnicas (como por exemplo descobertas de novos algoritmos, estruturas ou arquiteturas)?

1

2

3

4

5

Regulamente Freqüentemente As vezes Raramente Nunca

2. Seu trabalho oferece oportunidades em desenvolvimento profissional (tais como aprendizagem e descobertas)

12

3

45

Regulamente Freqüentemente As vezes Raramente Nunca

3. Como seu trabalho se apresenta a você :

$\begin{array}{llllll}\text { Excitante/ Monótono } & 1 & 2 & 3 & 4 & 5 \\ \text { Rotineiro /Inventivo } & 1 & 2 & 3 & 4 & 5 \\ \text { Maçante / Desafiador } & 1 & 2 & 3 & 4 & 5\end{array}$

4. Sua empresa tem um adequado programa de recompensa (prêmios, desempenho, produtividade)?

$$
\begin{aligned}
& \text { 1-Sim, muito generoso 2-Sim, generoso } 3 \text { - Sim, } \\
& \text { justo } \\
& 4 \text { - Sim, mas injusto 5- Não }
\end{aligned}
$$

5. Avalie objetivamente as oportunidades para progresso de sua carreira em sua empresa.

$$
\begin{array}{lll}
1 \text { - Excelentes } & 2 \text { - Boas } & 3 \text { - Médias } \\
4 \text { - Ruins } & 5 \text { - Muito ruins }
\end{array}
$$

6. Avalie objetivamente a supervisão técnica que você encontra na sua empres a.

$$
\begin{array}{lll}
1 \text { - Excelentes } & 2 \text { - Boas } & 3 \text { - Médias } \\
4 \text { - Ruins } & 5 \text { - Muito ruins }
\end{array}
$$

7. Como você avalia suas responsabilidades técnicas ou administrativas

$$
\text { Insignificantes/ Importantes. } \quad \begin{array}{lllllll}
1 & 2 & 3 & 4 & 5
\end{array}
$$

8. Avalie sua relação com seus colegas de trabalho.
1 - Amigável
2 - Competitiva
3 - Produtiva 
9 Avalie sua relação com seus subordinados Antagônica

$$
1 \text { - Amigável produtiva }
$$

3 - Estritamente profissional
2 - Produtiva

4 - Antagônica
5 - Muito

10 Como você compara seu salário com respeito a seu esforço e habilidade?

$\begin{array}{lllllll}\text { Baixo/alto } & 1 & 2 & 3 & 4 & 5\end{array}$

11 Com que frequêencia seu trabalho interfere na sua vida pessoal (hora extra, viagens, trabalho levado para casa, etc. )?

$$
\begin{array}{ll}
1 \text { - Regularmente } & 2 \text { - Freqüentemente } \\
3 \text { - Às vezes } & 4 \text { - Raramente }
\end{array}
$$

12 Avalie sua relação com seu superior.

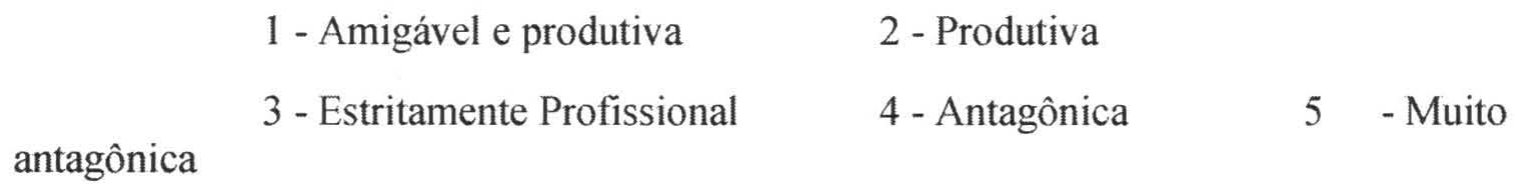

13 Quanto você se sente seguro no seu trabalho?

Dispensável

$$
1 \text { - Estável 2-Estabilizando-se }
$$$$
3 \text { - Instável, mas indispensável } 4 \text { - |Instável } \quad 5 \quad \text { - }
$$

14 Avalie quanto prestígio você alcança em sua empresa em relação as suas responsabilidades e tarefas.

Baixo

$$
\begin{array}{lcc}
1 \text { - Muito alto com relação a posição e responsabilidade } & 2 \text {-Alto } \\
3 \text { - Adequado } & 4 \text { - Baixo } & 5 \text { - Muito }
\end{array}
$$

15 Você concorda com a política da empresa (em termos de estrutura hierárquica, compartilhamento de poder e tomada de decisão)?

Adequada/Inadequada

$\begin{array}{lllll}1 & 2 & 3 & 4 & 5\end{array}$

16 Avalie suas condições de trabalho.
1 -Excelente
2 - Boa
3 - Média 